\title{
Improvement of Hyperlipidemia by Aerobic Exercise in Mice through a Regulatory Effect of miR-21a-5p on its Target Genes
}

Jinfeng Zhao

Shanxi University

Yaxin Wang

Beijing Sport University

Anping Chen

Shanxi University

Yu Zeng

Shanxi University

Feng Yan

Shanxi University

Longchang Chen

Shanxi University

Baoai Wu ( $269203910 @ q q . c o m)$

Shanxi University

\section{Research Article}

Keywords: Hyperlipidemia , Aerobic exercise , MiR-21a-5p , Target gene

Posted Date: January 13th, 2021

DOl: https://doi.org/10.21203/rs.3.rs-132701/v1

License: (9) (i) This work is licensed under a Creative Commons Attribution 4.0 International License.

Read Full License 


\section{Abstract}

Background and aims Hyperlipidemia is a risk factor for cardiovascular disease, and miR-21a-5p plays an important role in the occurrence and progression of hyperlipidemia, this study aimed to investigate the mechanism of aerobic exercise improved hyperlipidemia through enhancement miR-21a-5p expression.

Method Spectrophotometer detected blood lipid level; HE stained liver tissue to check the grade of liver steatosis; QPCR and Western blot detected mRNA expression and protein levels.

Results There had a notable improvement in the weight gain, blood lipid level and liver steatosis in hyperlipidemia mice after 8 weeks aerobic exercise intervention. In addition, aerobic exercise significantly up-regulated the expression of miR-21a-5p, and provoked favorable changes in the expression of target genes. Knockdown of miR-21a-5p resulted in dysregulation of lipid metabolism and increased expression of target genes FABP7, HMGCR, ACAT1 and OLR1. While aerobic exercise could alleviate miR-21a-5p knock-down induced lipid metabolism disorder.

Conclusion Aerobic exercise improved hyperlipidemia through miR-21a-5p-induced inhibition of target genes FABP7, HMGCR, ACAT1 and OLR1.

\section{Introduction}

Hyperlipidemia is characterized by elevated levels of plasma triglycerides and cholesterol. However, disorders of lipid metabolism are risk factors for cardiovascular diseases such as atherosclerosis, stroke and coronary heart disease ${ }^{1}$.

A growing body of evidence suggests that non-coding RNAs, such as Inc-RNA, microRNA, contributes to the development of hyperlipidemia. MiRNA as an important target for regulating lipid metabolism and homeostasis has been a hot topic in recent years ${ }^{2}$. MicroRNAs (miRs) are small non-coding RNAs consisting of 20-22 nucleotides (nt) that interact with short motifs in the $3^{\prime}$ untranslated region (UTR) of target genes, causing translational repression and/or mRNA destabilization ${ }^{3}$. MiR-21a is one of the first mammalian microRNAs identified, which involves in many physiological processes and relates to a variety of diseases, one among its most representative roles is to regulate lipid metabolism ${ }^{4}$. Many researches have demonstrated that the expression of miR-21a-5p was downregulated in nonalcoholic fatty liver patients or high-fat fed mice and miR-21a-5p knock-out can lead to hepatic steatosis, which accelerated atherosclerosis, plaque necrosis, and vascular inflammation. Thus, miR-21a-5p is a potential target for lipid metabolism regulation ${ }^{5}$.

MiR-21a-5p affects lipid metabolism mainly by regulating the expression of genes related to lipid homeostasis. A single miRNA has multiple targets, and simultaneously regulates target mRNAs involved in a physiological pathway ${ }^{6}$. In many studies, we have found that the target genes of miR-21a-5p involved in lipid metabolism, including fatty acid-binding protein $7(\mathrm{FABP} 7)^{7}$, 3-hydroxy-3-methylglutaryl- 
coenzyme A reductase $(\mathrm{HMGCR})^{5}$, peroxisome proliferator-activated receptor a (PPARa $)^{8}$ and phosphatase and tensin homolog deleted on chromosome $10(\mathrm{PTEN})^{9}$. In addition, bio-informatic prediction using TargetScan, miRTarBase revealed acetyl-CoA acetyltransferase 1 (ACAT1) and oxidized low density lipoprotein receptor 1 (OLR1) are potential targets of miR-21a-5p and closely relate to cholesterol metabolism.

As a non-pharmaceutical intervention, aerobic exercise can effectively prevent obesity, improve diabetes and cardiovascular disease, and many studies have demonstrated that aerobic exercise can reduced the pathogenesis and progression of hyperlipidemia through different processes. Maxi Meissner et al. suggested that voluntary wheel running ameliorates cholesterol metabolism, mainly by promoting its conversion into bile acids or decreasing intestinal cholesterol absorption ${ }^{10}$. Other researches showed that aerobic exercise can inhibit cholesterol synthesis by reducing the expression and activity of HMGCR ${ }^{11}$. In addition, our previous studies showed that aerobic exercise reduced the expression of PCSK9, an important gene for cholesterol metabolism, and enhanced LDLR expression ${ }^{12}$. Now some studies confirm that exercise could exert its beneficial effects in the improvement of lipid metabolism by the regulation of miRNA biology ${ }^{13,14}$, but the underlying mechanism of miRNA in mediating the protective effect of exercise against lipid accumulation remains largely unclear.

In this study, we focused on the role of miR-21a-5p in regulating lipid metabolism by affecting its target genes FABP7, PPARa, PTEN, HMGCR, ACAT1 and OLR1. And we want to know whether aerobic exercise can affect the expression of miR-21a-5p, so as to change the expression of its target genes and achieve the goal of lowering lipid accumulation.

\section{Materials And Methods \\ 2.1 Animal study}

SPF 8-week-old male C57BL/6J mice $(n=30)$ were provided by Beijing vital river laboratory animal technology biotech (Beijing, China) and fed in China institute for radiation protection (CIRP), the animal license number was SCXK (Beijing) 2016-0006, weighing $21.4 \pm 0.92 \mathrm{~g}$. After one-week adaptive feeding, the mice were randomly divided into 3 groups, 10 in each group. Mice in the NC group were fed a chow diet, $\mathrm{HH}$ group were fed a high-fat/ high-cholesterol diet, and HHE group received aerobic exercise intervention for 8 weeks on the basis of high-fat/ high-cholesterol diet (Fig. 1). Raising condition: temperature was $20-26^{\circ} \mathrm{C}$, humidity was $40 \%-60 \%$, light and dark cycle each 12 hours, all mice had free access to tap water and food. After the final exercise, the mice fasted overnight and then sacrificed. Blood samples were collected in tubes containing EDTA and centrifuged at $3500 \mathrm{rpm}$ at $4{ }^{\circ} \mathrm{C}$ for $15 \mathrm{~min}$, and the plasma was transferred to new tubes, stored at $-80^{\circ} \mathrm{C}$. Mice livers were quickly removed, snap frozen in liquid nitrogen after washed with cold phosphate buffered saline (PBS), stored at $-80^{\circ} \mathrm{C}$ for future analysis. 
Anti-miR-21a-5p adeno-associated virus vector (AAV) $\left(100 \mu \mathrm{L}, 1.95 \times 10^{\wedge} 12 \mathrm{vg} / \mathrm{ml}\right)($ Sangon biotech, Shanghai, China) was injected into the tail vein to specifically inhibit the expression of miR-21a-5p in the liver $(n=10)$, and empty vector was served as negative control $(n=10)$. Five weeks after injection, miR$21 a-5 p$ KD mice on chow diet or high-fat diet were given a 5 weeks aerobic exercise intervention, then mice were sacrificed after the last exercise. All animal studies were conducted in accordance with the Ethics Committee of Scientific Research in Shanxi University, experiments conformed to local and international guidelines on ethical use of animals, and this study adhered to the ARRIVE guidelines.

\subsection{Exercise program}

After four weeks of high fat / high cholesterol feeding, HHE groups underwent a one-week treadmill exercise training, followed by 8 weeks formal aerobic exercise. The intensity of aerobic exercise was determined to be $18 \mathrm{~m} / \mathrm{min} \times 45 \mathrm{~min}, 6 \%$ slope, 6 day/week, and each exercise had a 5 -minute warm-up, total 8 weeks.

\subsection{Lipid Profile}

Serum total cholesterol (TC), triglyceride (TG), LDL cholesterol (LDL-C), HDL cholesterol (HDL-C) was assayed by spectrophotometer (UV-6100s, Mapada, Shanghai, China) according to the kit's instructions (Nanjing jian cheng biotech, China). The content of TG and TC were measured at $510 \mathrm{~nm}, \mathrm{LDL}-\mathrm{C}$ and HDL-C were measured at $546 \mathrm{~nm}$.

\subsection{Histologic analysis}

Liver tissues were fixed in 10\% neutral buffered formalin, then sections were stained with standard hematoxylin-eosin (H\&E), the degree of liver steatosis was observed under a microscope.

\subsection{Real time Quantitative RT-PCR}

Total RNA was isolated from mice liver sample using a spin column animal total RNA purification kit (Sangon biotech, Shanghai, China) in accordance with the manufacturer's instructions. M-MuLV firststrand cDNA synthesis kit was used for reverse transcription into CDNA with total RNA as template. MiRNA was isolated with a miRNeasy Mini kit and reverse transcription was performed using the Tailadded reverse transcription kit (Sangon biotech, Shanghai, China). Quantitative PCR was performed on a LightCycler480 system (Roche, Switzerland) using TB Green premix Ex Taq $\otimes$ mix (TaKaRa, Dalian, China), gene specific primers were listed in Table 1, $\beta$-actin and U6 small nuclear RNA (snRNA) was used as an internal control. 
Table 1

Gene-specific primers

\begin{tabular}{|c|c|c|}
\hline \\
\hline Gene & Direction & Sequence( $\left.5^{\prime}-3^{\prime}\right)$ \\
\hline \multirow[t]{2}{*}{ FABP7 } & Forward & GAAACCAGCATAGATGACAGAA \\
\hline & Reverse & TAACAGCGAACAGCAACGATA \\
\hline \multirow[t]{2}{*}{ PPARa } & Forward & TACTGCCGTTTTCACAAGTGC \\
\hline & Reverse & AGGTCGTGTTCACAGGTAAGA \\
\hline \multirow[t]{2}{*}{ HMGCR } & Forward & TCTGGCAGTCAGTGGGAACTATT \\
\hline & Reverse & CCTCGTCCTTCGATCCAATTT \\
\hline \multirow[t]{2}{*}{ PTEN } & Forward & TGGATTCGACTTAGACTTGACCT \\
\hline & Reverse & GCGGTGTCATAATGTCTCTCAG \\
\hline \multirow[t]{2}{*}{ ACAT1 } & Forward & CAGGAAGTAAGATGCCTGGAAC \\
\hline & Reverse & TTCACCCCCTTGGATGACATT \\
\hline \multirow[t]{2}{*}{ OLR1 } & Forward & CAAGATGAAGCCTGCGAATGA \\
\hline & Reverse & ACCTGGCGTAATTGTGTCCAC \\
\hline \multirow[t]{2}{*}{ Dicer1 } & Forward & GGTCCTTTCTTTGGACTGCCA \\
\hline & Reverse & GCGATGAACGTCTTCCCTGA \\
\hline miR-21a-5p & Forward & CCGCGTAGCTTATCAGACTGATGTTGA \\
\hline miR-33a & Forward & GTGCATTGTAGTTGCATTGCA \\
\hline miR-34 & Forward & AATCAGCAAGTATACTGCCCT \\
\hline miR-29a & Forward & TAGCACCATCTGAAATCGGTTA \\
\hline miR-27 & Forward & AGTTCACAGTGGCTAAGTTCCGC \\
\hline miR-200c & Forward & CCGTAATACTGCCGGGTAATGATGGA \\
\hline miR-122 & Forward & TGGAGTGTGACAATGGTGTTTG \\
\hline
\end{tabular}

\subsection{Western blot}

Mice liver tissues were lysed in RIPA lysis buffer, total soluble protein was quantified using the BCA Protein Assay kit (Beyotime, Shanghai, China). Proteins $(40 \mu \mathrm{g})$ were separated by sodium dodecyl sulfate polyacrylamide gel electrophoresis (SDS-PAGE) and transferred to PVDF membranes, blocked for 1 hour with $5 \%$ nonfat milk. After overnight incubation with primary antibody (1:1000 anti-FABP7, antiHMGCR, anti-PTEN, anti-PPARa, anti-ACAT1, anti-OLR1) (Proteintech Group Inc, Wuhan, China), membranes were washed and incubated with HRP-conjugated secondary antibody (1:5000) (Boster 
Biotech, Wuhan, China). Then the signals were detected using an ECL detection kit (Applygen Technologies Inc, Beijing, China) and imaged in a chemiluminescence-measuring instrument (ChemiDoc XRS+, Bio-Rad, USA).

\subsection{Statistical analysis}

All the experimental data were presented as mean \pm standard error (SD). Comparisons were performed with Student's t-test or One-way ANOVA followed by Tukey's post hoc test. Analysis program used SPSS (25.0). P-values were considered significant at $<0.05$.

\section{Results}

\subsection{Aerobic exercise beneficially affect body weight and plasma lipoprotein profile}

A high-fat/ high-cholesterol diet induced hyperlipidemia mouse model was used to evaluate the lipidlowering effects of aerobic exercise. As can be clearly seen from Fig. 2a, the weight of $\mathrm{HH}$ group mice showed a rapidly rising trend, significantly higher than that of the NC group at the end of the experiment $(P<0.05)$. However, from 6 weeks to 13 weeks of aerobic exercise intervention, the body weight of the HHE group was significantly diminished than HH group. In addition, after 12 weeks of highfat /high-cholesterol diet, fasting plasma lipids were significantly increased in HH mice than NC group, representing prominent serum lipids accumulation. While the serum lipid levels of $\mathrm{HH}$ group mice recovered significantly when underwent the aerobic exercise intervention for 8 weeks, indicated that aerobic exercise provoked favorable changes in plasma lipid level. (Fig. 2b).

\subsection{Aerobic exercise improved steatosis of high fat/high cholesterol diet mice}

The livers of the NC group were bright red with smooth surface and sharp edges by visual inspection, while the livers of the $\mathrm{HH}$ group were yellowish-brown with dull edges, we can make a preliminary judgement that the livers were covered with lipids after the high-fat/high cholesterol diet (Fig. 2c). Histologic analysis by H\&E staining displayed that the liver of hyperlipidemia mice revealed severe histopathological alterations described as numerous small cytoplasmic vacuoles associated with punctate necrosis of liver cells and infiltration of inflammatory cells. In contrast, we found that after aerobic exercise intervention, the number of liver lipid droplets were decreased and the size became smaller, the arrangement of liver cells was neat. (Fig. 2d)

\subsection{Aerobic exercise beneficially regulated the expression miR-21a-5p and its target genes}

Numerous miRNAs have been reported to involved in lipid regulating. In our previous experiment, we have detected the expression of miR-33, miR-34a, miR-21a, miR-29a, miR-27a, miR-200c, miR-122, the results 
showed that these miRNAs had a significantly different expression in $\mathrm{HH}$ group, and there was a notable diminished of miR-21a-5p (pष0.01)(Fig. 3a), which was consistence with other studies that miR-21a-5p expression was decreased in patients with NAFLD or mice on a high-fat diet. Whereas, after 8 weeks of aerobic exercise intervention, miR-21a-5p was significantly elevated (p囚0.05) (Fig. 3b). In addition, we detected the expression of Dicer1, an enzyme for miRNA processing, was decreased in HH group, but highly elevated in HHE group (Fig. 3c).

Previous studies have confirmed that FABP7, HMGCR, PPARa and PTEN are direct targets of miR-21a-5p. In addition, we predicted other target genes of miR-21a-5p by TargetScan and found that ACAT1 and OLR1 have a putative binding site in its 3'untranslated region (UTR) (Fig. 3d). To determine whether the target genes of miR-21a-5p responded to aerobic exercise, we measured their mRNA levels after aerobic exercise. Dates revealed that the expression of PPARa, PTEN declined in the HH group, but significantly increased after exercise intervention. On the contrary, the mRNA levels of FABP7, HMGCR, ACAT1 and OLR1 elevated significantly in the HH group, but at 8 weeks post-exercise intervention, all downregulated $(P<0.05)$ (Fig. 3e). And we also detected the protein levels of miR-21a-5p targets, which were consistent with mRNA level (Fig. 3f).

Among them, FABP7, PPARa, and PTEN are closely related to fat metabolism, and HMGCR, ACAT1 and OLR1 can regulate cholesterol synthesis and metabolism. Thus, we believed that one of the mechanisms that aerobic exercise improves hyperlipidemia may be by influencing the expression of genes related to lipid metabolism.

\section{4 miR-21a-5p regulated the expression of genes involved in lipid metabolism}

To further examine the impact of miR-21a-5p on hepatic steatosis and measure the effect of miR-21a-5p on its target genes, we specifically inhibited miR-21a-5p in mice liver using an AAV encoding anti-miR$21 \mathrm{a}-5 \mathrm{p}$. In order to detect the specific inhibitory effect, we examined the expression of miR-33, miR-34, miR-27 and miR-21a-5p, and found that compared with the control group, miR-27, miR-33, miR-34a had no obviously changes, the expression of miR-21a-5p decreased significantly (Fig. 4a).

miR-21a-5p KD mice on chow diet or high-fat diet showed increased body weight and serum lipid levels $(P<0.05)$ (Fig. 4a, b), these results indicated the mechanisms of lipid metabolism in vivo were impaired, resulting in lipid accumulation. Next, we verified the relationship between the expression of FABP7, PPARa, HMGCR, PTEN, ACAT1, OLR1 and miR-21a-5p through quantified the expression of these targets in miR-21a-5p KD mice. we can see that inhibition of miR-21a-5p significantly increased the FABP7, HMGCR, ACAT1 and OLR1 mRNA levels, while PPARa, PTEN had not significant changes (Fig. 4c). Therefore, we believed that miR-21a-5p regulates lipid metabolism mainly by affecting the expression of FABP7, HMGCR, ACAT1 and OLR1.

\subsection{Rescue effect of aerobic exercise on miR-21a-5p KD mice}


The fact that aerobic exercise can effectively reduce lipid accumulation has been proved, and the relationship between miR-21a-5p and lipid metabolism was well established, hence, it was essential to focus on whether the increased miR-21a-5p levels induced by aerobic exercise affect lipid accumulation, we conducted a five-week aerobic exercise intervention on miR-21a-5p KD mice with chow diet or high-fat diet and found that the expression of miR-21a-5p was regained after aerobic exercise (Fig. 5a). Moreover, we found that the blood lipid level and liver steatosis of miR-21a-5p KD mice were significantly improved (Fig. 5b, c), indicating that aerobic exercise could alleviated the lipid accumulation caused by the loss of miR-21a-5p, these results strongly verified that the involvement of miR-21a-5p in the improvement of hyperlipidemia by aerobic exercise. In addition, we found that aerobic exercise can reduce the mRNA levels of FABP7, HMGCR, ACAT1 and OLR1 in miR-21KD mice $(P<0.05)$ (Fig. 5d). Therefore, we showed that one of the mechanisms by which aerobic exercise regulates lipid metabolism was to up-regulate the expression of miR-21a-5p, thereby suppressing the expression of its target genes FABP7, HMGCR, ACAT1 and OLR1.

\section{Discussion}

In our experiment, the weight gain, plasma lipid levels and liver pathology of high fat/high cholesterol diet mice had a favorable shift after 8 weeks aerobic exercise. And we think one of the mechanisms by which aerobic exercise improved hyperlipidemia was to up-regulate the expression of miR-21a-5p, thus inhibited the expression of target genes FABP7, HMGCR, ACAT1 and OLR1, which were closely related to lipid metabolism.

MiR-21a-5p plays a key role in suppressing the occurrence and development of hyperlipidemia, we have shown that the expression of miR-21a-5p had a significant reduction in hyperlipidemia mice, and inhibition of miR-21a-5p would expect to result in a disorder of lipid metabolism. Whereas, the opposite was observed after aerobic exercise intervention. Recent studies have demonstrated that exercise improves non-alcoholic fatty liver disease, atherosclerosis, cancer and other diseases by regulating miRNAs. Xiao J. J et al provided compelling evidence suggesting that miR-212 might be a novel therapeutic target mimicking the benefit of exercise in the treatment of NAFLD ${ }^{14}$. In addition, aerobic exercise and statins can induce the expression of miR-146a, thereby reducing the TRAF and TLR4 signaling pathways and vascular inflammatory damage in atherosclerosis, confirming the protective effect of aerobic exercise on vascular diseases ${ }^{15}$. Furthermore, exercise could effectively reduce the tumor volume, lower levels of proinflammatory cytokines TNFa, increase the anti-inflammatory cytokine IL-10 by regulating miR-21a-5p expression ${ }^{16}$. Taken together, these researches indicated that aerobic exercise could play a variety of physiological regulatory roles through miRNA, and miR-21a-5p is an important target for exercise to improve lipid metabolism.

As we all know Dicer1 is essential for miRNA maturation, the disruption of the Dicer1 results in the loss of mature miRNAs. Ming-xia LIU et al. have shown that the expression of Dicer1 was significantly reduced in NASH mice model and inversely associated with hepatic FC level. Through further research they found that serum lipid disorder caused by Dicer1 deletion may be due to the decreased expression of miR-29, 
which improve nonalcoholic fatty liver disease by suppressing HMGCR expression ${ }^{17}$. This study made us wonder aerobic exercise increased the expression of miR-21a-5p may be through the enhancement of Dicer1 expression. Interestingly, Aaron P Russell et al. have found that after $60 \mathrm{~min}$ acute endurance exercise, miRNA processing complex, including Drosha, Dicer and Exportin-5, were significantly upregulated by 35,35 and $30 \%$, respectively ${ }^{18}$. And our study also showed that Dicer1 expression decreased in the $\mathrm{HH}$ group, while increased after exercise intervention, which may be one of the reasons why exercise promoted miR-21a-5p expression.

Researches on mechanism of miRNAs regulate target gene expression are generally believed that miRNA is to induce post-transcriptional gene silencing through mRNA degradation or translation repression.

Mihnea et al summarized seven unconventional ways in which miRNAs can exert regulatory functions ${ }^{19}$, and recently the function of miRNA has been extended to transcriptional levels, either directly or indirectly ${ }^{20}$. In our study, the expression of FABP7, HMGCR, ACAT1 and OLR1 were increased in miR-21a$5 p$ KD mice, indicated that miR-21a-5p played a negative regulatory role on these target genes, whether this negative regulatory effect is at the post-transcriptional level or at the transcriptional level still needs further in-depth study. Importantly, recent studies have indicated that miRNAs have a dual role. When it is located in the cytoplasm, miRNAs inhibit gene expression, when it is in the nucleus, it can activate gene expression by binding to the target gene enhancer ${ }^{21}$. If the miRNA is distributed in both the cytoplasm and the nucleus, then it is likely to perform multiple functions on the target genes. And researches have revealed that miR-21a-5p could be detected both in the cytosol and in the nucleus ${ }^{22}$, so we will pay more attention to how miR-21a-5p regulates the expression of target genes in the nucleus in the future.

In addition, in our study, it was found that there was no significant change in PTEN and PPARa in miR$21 a-5 p$ KD mice, the result might reflect a compensatory mechanism of the organism under the stimulation of pathological stress of dyslipidemia after miR-21a-5p knockdown. Then, we thought that there may be other pathways were implicated in the regulation of aerobic exercise on the expression of PTEN and PPARa other than miR-21a-5 $\mathrm{p}^{23}$.

In conclusion, this study provided new insights into the mechanism of aerobic exercise regulated lipid metabolism through miRNA. Our work identified miR-21a-5p as a potential regulator of aerobic exercise affecting lipid metabolism, achieved the favorable goal in hyperlipidemia by synergistically inhibiting the expression of target genes FABP7, HMGCR, ACAT1, and OLR1.

\section{Declarations}

\section{Acknowledgement}

This research was supported by the National Natural Youth Science Foundation of China (grant number 31500962). 


\section{Declaration of competing interest}

The authors declare that they have no conflict of interest.

\section{Author contributions}

Jinfeng zhao: Conceptualization, Methodology, Investigation, Formal analysis, Writing - original draft, Writing-review \&amp; editing. Yaxin Wang: Conceptualization, Methodology, Investigation, Writing original draft. Anping Chen: Writing-review \& editing. Yu Zeng: Investigation, Writing-review \& editing. Feng Yan: Investigation, Writing-review \& editing. Longchang Chen: Investigation, Writing-review \& editing. Baoai Wu: Conceptualization, Formal analysis, Writing-review \&amp; editing.

\section{References}

1. Navar-Boggan, A. M. et al. Hyperlipidemia in Early Adulthood Increases Long-Term Risk of Coronary Heart Disease. Circulation. 131, 451-458 https://doi.org/10.1161/CIRCULATIONAHA.114.012477 (2019).

2. Bartel, D. P. \& MicroRNAs Target recognition and regulatory functions. Cell. 136, 215-233 https://doi.org/10.1016/j.cell.2009.01.002 (2009).

3. Churov, A., Summerhill, V., GrechKD, A., Orekhova, V. \& Orekhov, A. MicroRNAs as Potential Biomarkers in Atherosclerosis. Int J Mol Sci. 20, 5547 https://doi.org/10.3390/ijms20225547 (2019).

4. Krichevsky, A. M. \& Gabriely, G. MiR-21: a small multi-faceted RNA. J Cell Mol Med. 13, https://doi.org/10.1111/j.1582-4934.2008.00556.x (2009).

5. Sun, C. Z. et al. MiR-21 regulates triglyceride and cholesterol metabolism in non-alcoholic fatty liver disease by targeting HMGCR. Int J Mol Med. 35, 847-853 https://doi.org/10.3892/ijmm.2015.2076 (2015).

6. Hanin, G. et al. MiRNA-132 induces hepatic steatosis and hyperlipidaemia by synergistic multitarget suppression. Gut. 67, 1124-1134 https://doi.org/10.1136/gutjnl-2016-312869 (2018).

7. Ahn, J., Lee, H., Jung, C. H. \& Ha, T. Lycopene inhibits hepatic steatosis via microRNA-21 induced downregulation of fatty acid-binding protein 7 in mice fed a high-fat diet. Mol Nutr Food Res. 56, 1665-1674 https://doi.org/10.1002/mnfr.201200182 (2012).

8. Rodrigues, P. M. et al. MiR-21 ablation and obeticholic acid ameliorate nonalcoholic steatohepatitis in mice. Cell Death Dis. 2017, 8, e2748; 10.1038/cddis.2017.246 (2017).

9. Liu, H. F. et al. Long Noncoding RNA GAS5 Suppresses 3T3-L1 Cells Adipogenesis Through miR-21a5p-5p/PTEN Signal Pathway. DNA Cell Biol. 37, 767-777 https://doi.org/10.1089/dna.2018.4264 (2018).

10. Meissner, M. et al. Voluntary wheel running increases bile acid as well as cholesterol excretion and decreases atherosclerosis in hypercholesterolemic mice. Atherosclerosis. 218, 323-329 
https://doi.org/10.1016/j.atherosclerosis.2011.06.040 (2011).

11. Li, J. W., Shang, Y., Ma, H. \& Ye, X. L. The Effect of Aerobic Exercise Combined with Rhizoma Coptidis Capsules on the Hypercholesterolemia Induced by as High-Fat and High-Cholesterol Diet in Syrian Golden Hamsters. Invest Clin. 60, 126-135 (2019).

12. Zhao, J. F. et al. Aerobic exercise combined with samara oil can improve hyperlipidemia by reducing PCSK9 and increasing LDLR. Intej clin Exp med. 12, 5191-5197 (2019).

13. Soci, U. P. et al. MicroRNAs 29 are involved in the improvement of ventricular compliance promoted by aerobic exercise training in rats. Physiol Genomics. 43, 665-673 https://doi.org/10.1152/physiolgenomics.00145.2010 (2011).

14. Xiao, J. J. et al. MiR-212 downregulation contributes to the protective effect of exercise against nonalcoholic fatty liver via targeting FGF-21. J Cell Mol Med. 20, 204-216 https://doi.org/10.1111/jcmm.12733 (2015).

15. Wu, X. D. et al. Effect of Aerobic Exercise on miRNA-TLR4 Signaling in Atherosclerosis. Int J Sports Med. 35, 344-350 https://doi.org/10.1055/s-0033-1349075 (2014).

16. Isanejad, A. et al. MicroRNA-206, Let-7 and microRNA-21 pathways involved in the anti-angiogenesis effects of the interval exercise training and hormone therapy in breast cancer. Life Sci. 151, 30-40 https://doi.org/10.1016/j.lfs.2016.02.090 (2016).

17. Liu, M. X. et al. Dicer1/miR-29/HMGCR axis contributes to hepatic free cholesterol accumulation in mouse non-alcoholic steatohepatitis. Acta Pharmacol Sin. 38, 660-671 https://doi.org/10.1038/aps.2016.158 (2017).

18. Russell, A. P. et al. Regulation of miRNAs in human skeletal muscle following acute endurance exercise and short-term endurance training. J Physiol. 591, 4637-4653 https://doi.org/10.1113/jphysiol.2013.255695 (2013).

19. Dragomir, M. P., Knutsen, E., Calin, G. A. \& SnapShot Unconventional miRNA Functions. Cell. 174, 1038-1038 https://doi.org/10.1016/j.cell.2018.07.040 (2018).

20. Pu, M. F. et al. MiR-1254 suppresses HO-1 expression through seed region-dependent silencing and non-seed interaction with TFAP2A transcript to attenuate NSCLC growth. Plos Genet. 13, e1006896 https://doi.org/10.1371/journal.pgen.1006896 (2017).

21. Xiao, M. et al. MicroRNAs activate gene transcription epigenetically as an enhancer trigger. RNA Biol. 14, 1326-1334 https://doi.org/10.1080/15476286.2015.1112487 (2017).

22. Meister, G. et al. Human Argonaute2 mediates RNA cleavage targeted by miRNAs and siRNAs. Mol Cell. 15, 185-197 https://doi.org/10.1016/j.molcel.2004.07.007 (2004).

23. Wang, D. W. et al. MicroRNA-20a participates in the aerobic exercise-based prevention of coronary artery disease by targeting PTEN. Biomed Pharmacother. 95, 756-763 https://doi.org/10.1016/j.biopha.2017.08.086 (2017).

\section{Figures}


Fig. 1

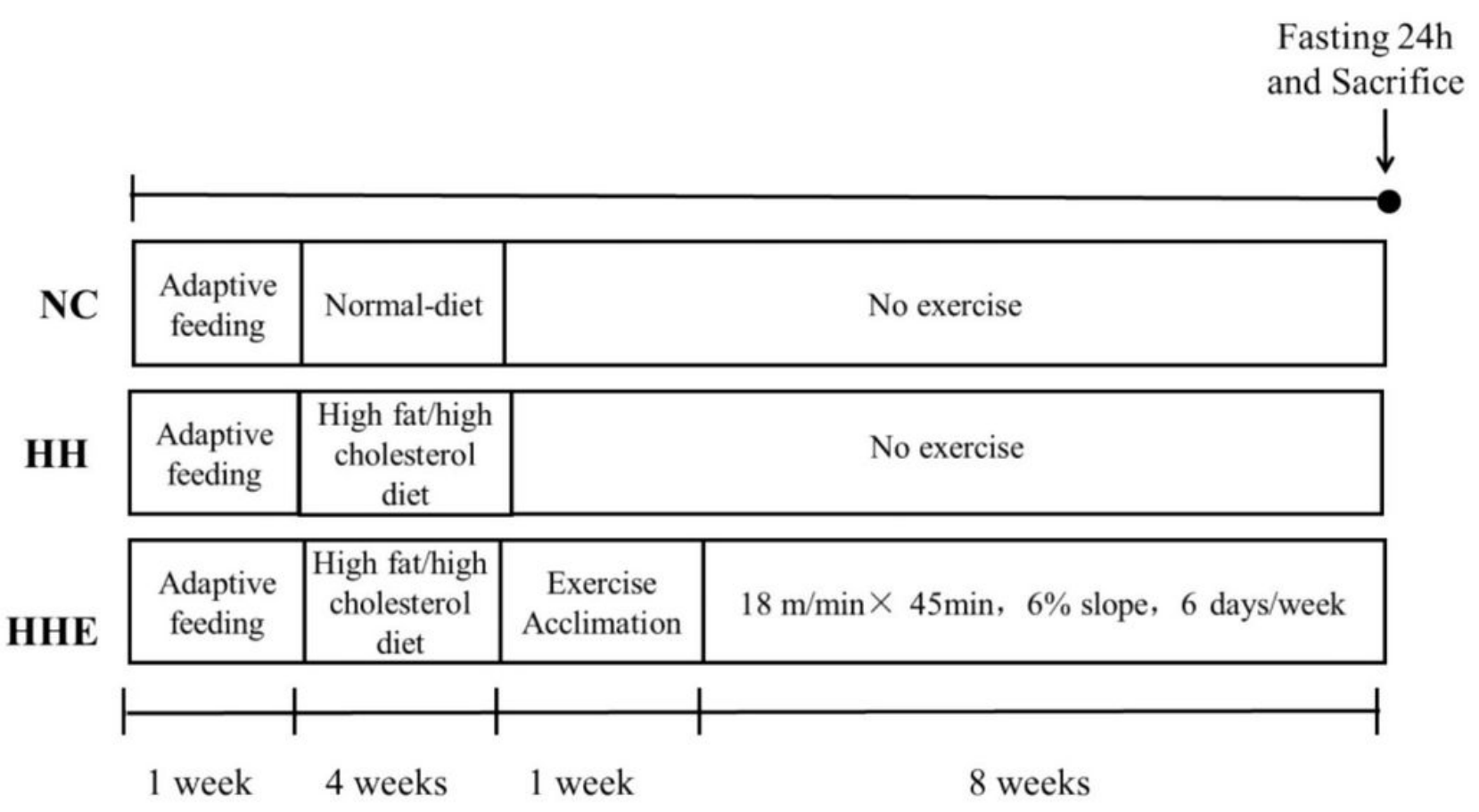

Figure 1

Design of the experiment. 
Fig. 2
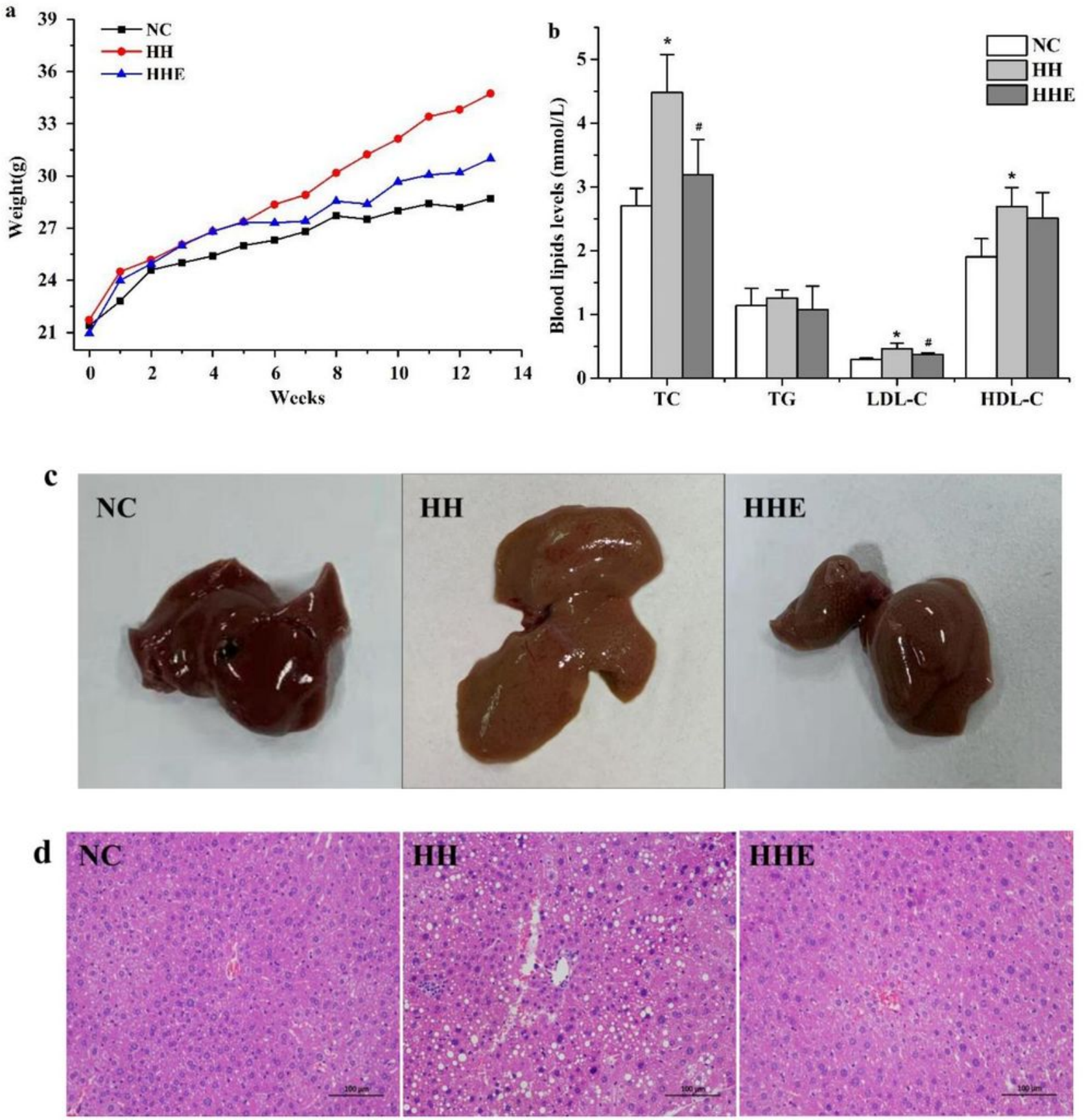

\section{Figure 2}

Protective effect of aerobic exercise on lipid metabolism disorders. (a) Trend of body weight change of mice in each group. (b) Serum lipids of mice in each group. (c) Visual observation of mice liver tissue. (d)

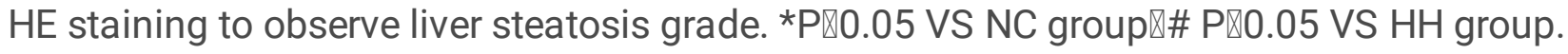



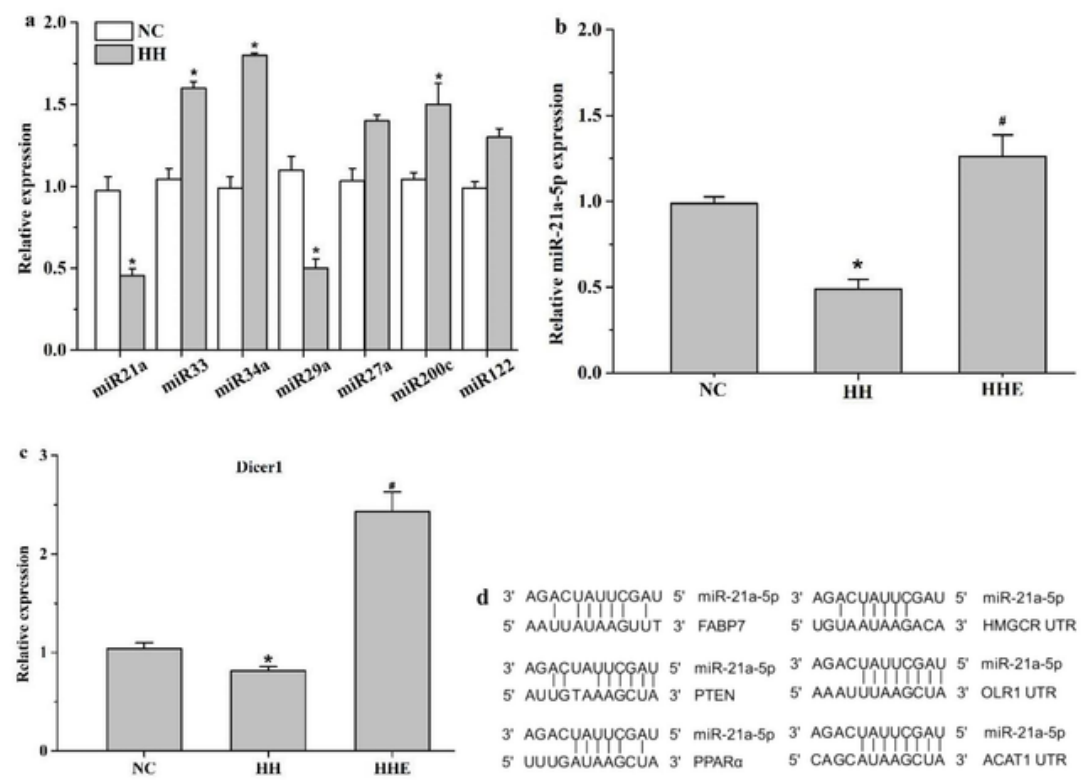

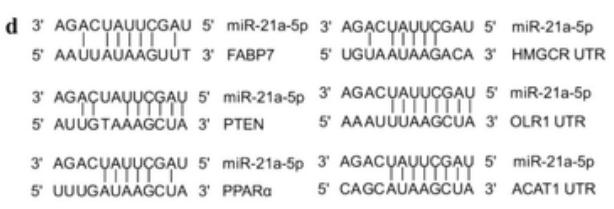
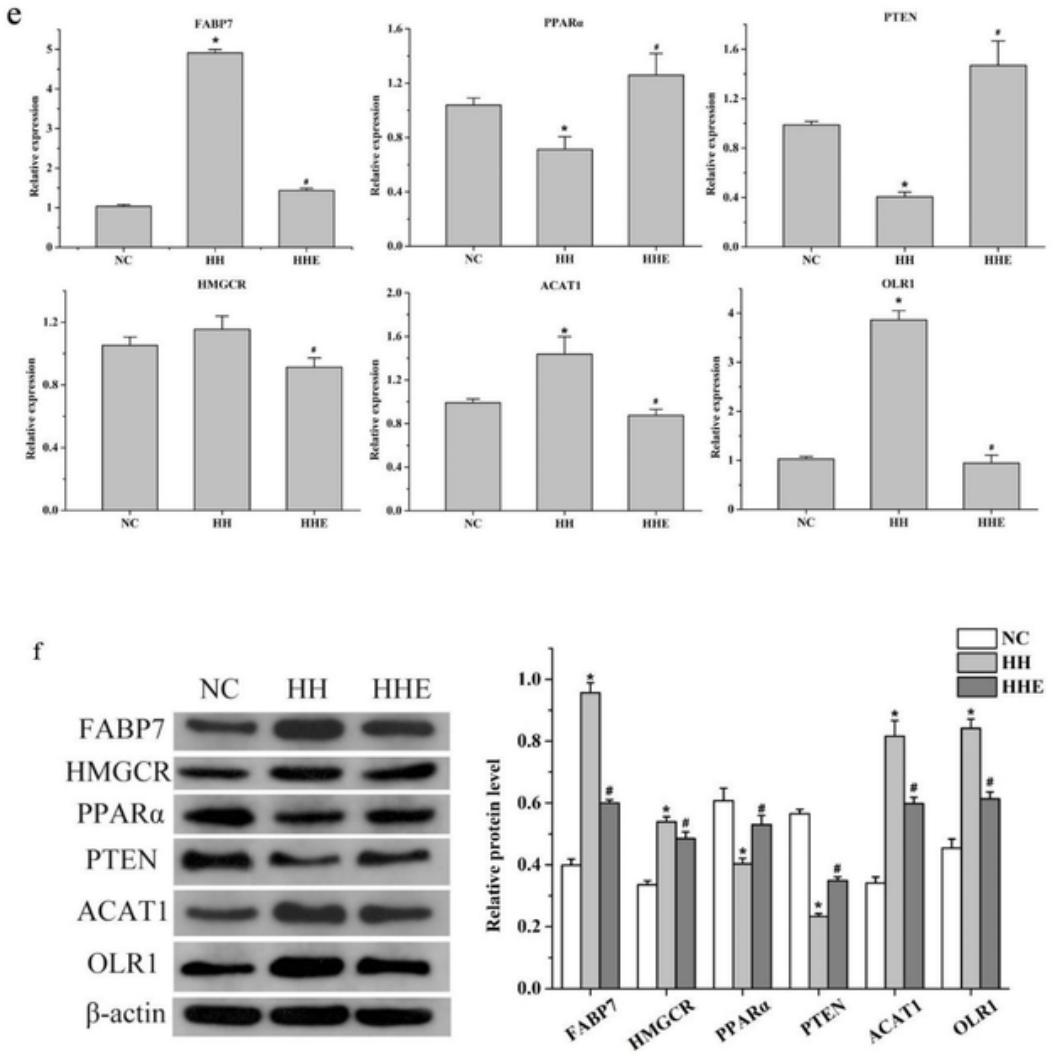

\section{Figure 3}

Effects of aerobic exercise on the expression of miR-21a-5p and its target genes. (a) Relative levels of miRNAs in liver of mice fed a high-fat diet. (b) QPCR was used to detect the expression of miR-21a-5p in $\mathrm{NC}, \mathrm{HH}$ and HHE groups. (c) The mRNA levels of Dicer1. (d) Schematic diagram of miR-21a-5p seed sequence with 3'-UTR binding site of target genes. (e) The mRNA levels of miR-21a-5p targets were detected by QPCR. (f) The protein levels of miR-21a-5p targets were determined by western blot, original 


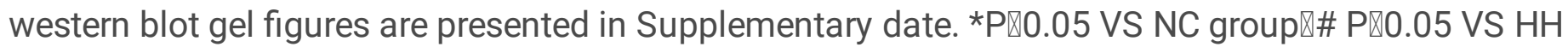
group.

Fig. 4
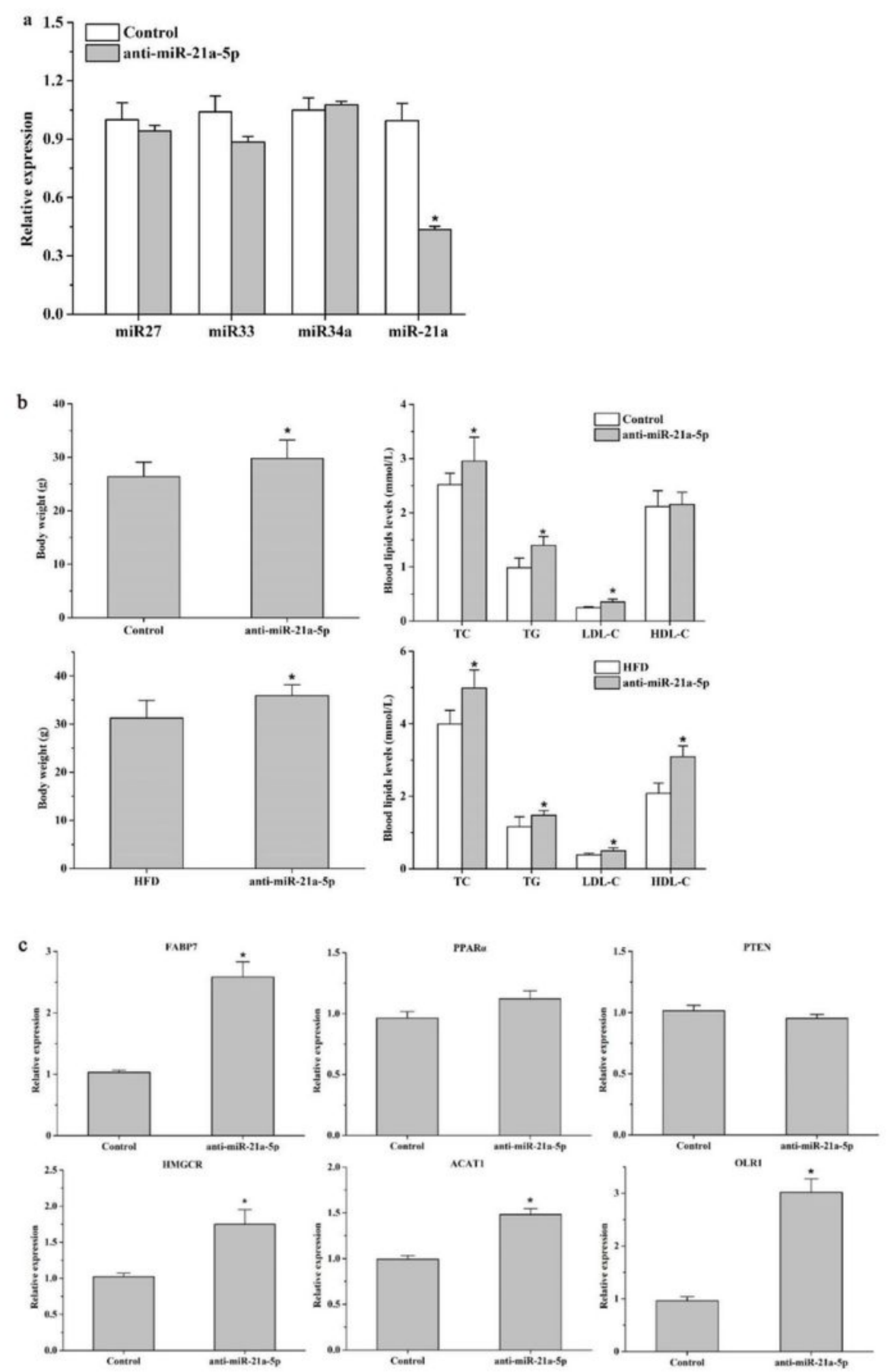

\section{Figure 4}

Knock-down of miR-21a-5p led to lipid metabolism disorder. (a) The expression of miR-27, miR-34a, miR33, miR-21a-5p after injected anti-miR-21a-5p AAV four weeks. (b) The body weight and serum lipid levels 
of miR-21a-5p KD mice on chow diet (c) mRNA levels of target genes in miR-21a-5p KD mice. *P『0.05 VS Control or HFD group

Fig. 5
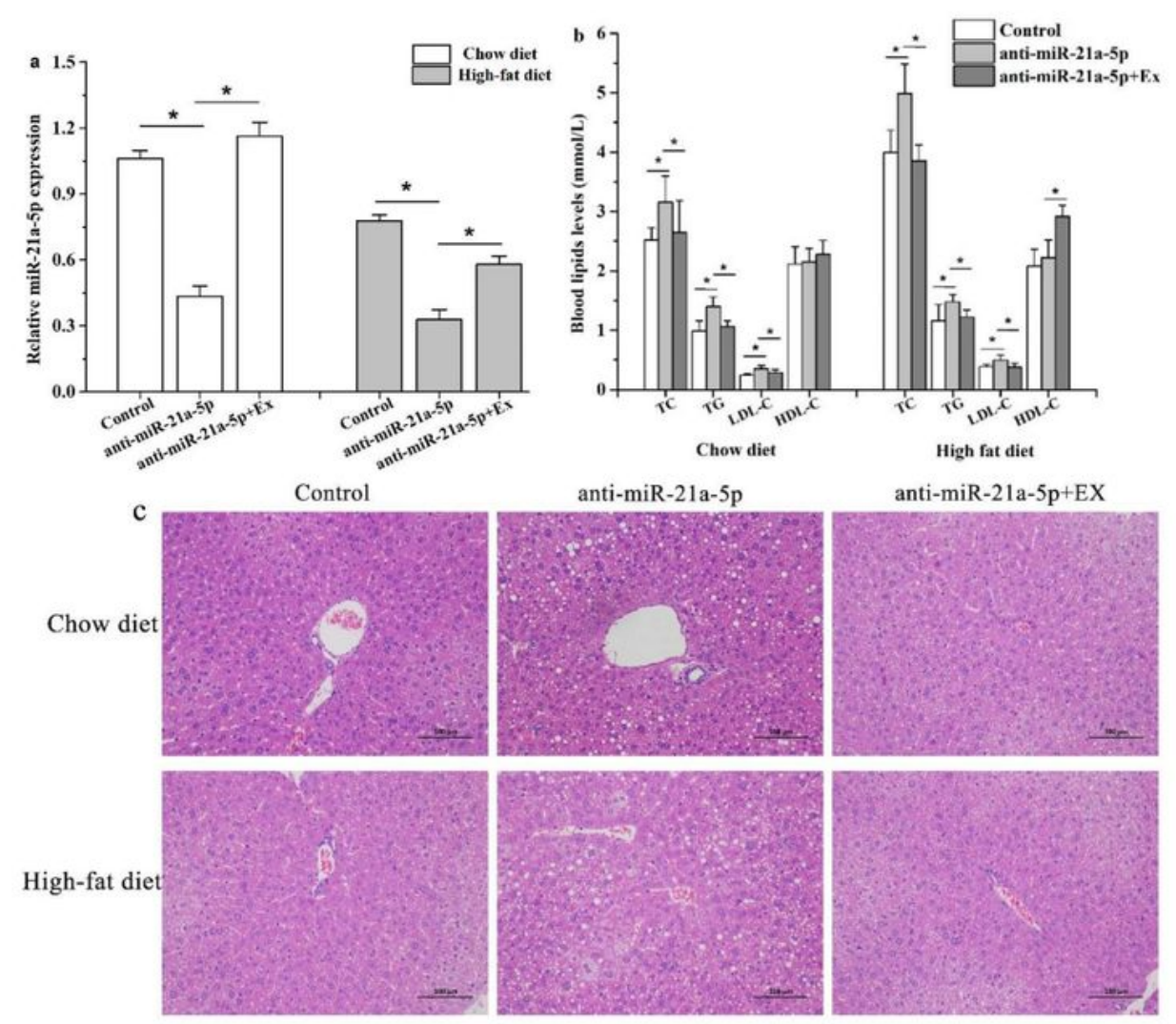

d
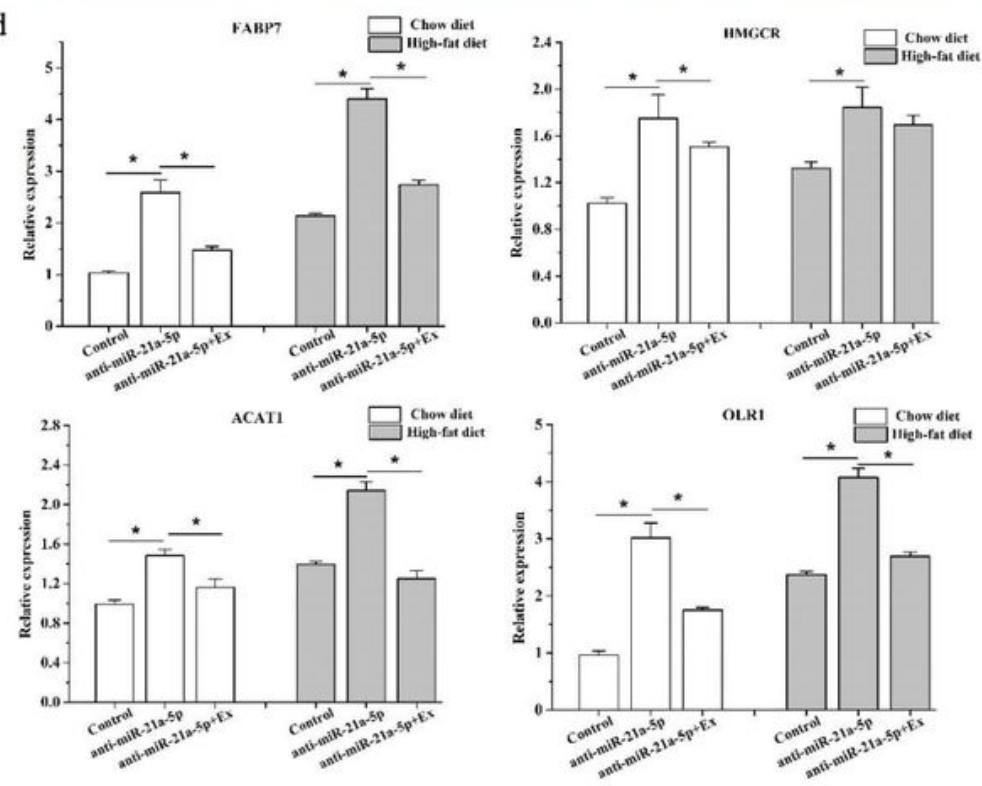

Figure 5

Improvement effects of aerobic exercise on miR-21a-5p KD mice with chow diet or high-fat diet. (a) The expression of miR-21a-5p (b) The serum lipid levels (c) H\&E staining to assess liver steatosis (d) The 
mRNA levels of target genes in miR-21a-5p KD mice after exercise intervention. *Pख0.05 VS Control group, \# P®0.05 VS anti-miR-21a-5p group.

\section{Supplementary Files}

This is a list of supplementary files associated with this preprint. Click to download.

- Supplementarydate.docx 